\title{
Endovascular treatment for type A aortic dissection - What are our critical concerns?
}

\author{
Ting Wei Chang ${ }^{1,2}$, Ting Ting Yang ${ }^{3}$, Hong Jie Jhou ${ }^{1,4}$, Liang Ying Ke ${ }^{1}$, Ying Fu Chen ${ }^{5,6,7}$ \\ ${ }^{1}$ School of Medicine, Kaohsiung Medical University, Kaohsiung 807, Taiwan. \\ ${ }^{2}$ Department of Surgery, Kaohsiung Medical University Hospital, Kaohsiung Medical University, Kaohsiung 807, Taiwan. \\ ${ }^{3}$ School of Post-baccalaureate Medicine, Kaohsiung Medical University, Kaohsiung 807, Taiwan. \\ ${ }^{4}$ Division of General Practice, Department of Medical Education, Changhua Christian Hospital, Changhua 500, Taiwan. \\ ${ }^{5}$ Division of Cardiovascular Surgery, Department of Surgery, Kaohsiung Medical University Hospital, Kaohsiung Medical \\ University, Kaohsiung 807, Taiwan. \\ ${ }^{6}$ Division of Cardiovascular Surgery, Tainan Sin-Lau Christian Hospital, Tainan 701, Taiwan. \\ ${ }^{7}$ Graduate Institute of Medicine, Kaohsiung Medical University, Kaohsiung 807, Taiwan.
}

Correspondence to: Dr. Ying Fu Chen, Division of Cardiovascular Surgery, Department of Surgery, Kaohsiung Medical University Hospital, Kaohsiung Medical University, No. 100, Tzyou First Road, Kaohsiung 807, Taiwan. E-mail: yfchen@kmu.edu.tw

How to cite this article: Chang TW, Yang TT, Jhou HJ, Ke LY, Chen YF. Endovascular treatment for type A aortic dissection - What are our critical concerns? Vesse/ P/us 2020;4:20. http://dx.doi.org/10.20517/2574-1209.2020.10

Received: 28 Mar 2020 First Decision: 20 Apr 2020 Revised: 23 May 2020 Accepted: 29 May 2020 Published: 12 Jul 2020

Academic Editor: Cristiano Spadaccio Copy Editor: Cai-Hong Wang Production Editor: Tian Zhang

\section{INTRODUCTION}

Endovascular graft intervention has brought new therapeutic concepts to conventional cardiovascular surgery. In 1991, an Argentinean team first described aortic endograft placement in the repair of abdominal aortic aneurysms, paving the way for new interventions in the management of aortic disease. Following this, endovascular aneurysm repair (EVAR) and thoracic EVAR (TEVAR) were developed in succession ${ }^{[1]}$. In 1999, Dake et al ${ }^{[2]}$ reported promising results (100\% technical success and $16 \%$ 30-day mortality) in the endovascular management of patients with acute aortic dissection, including 4 patients with retrograde acute type $\mathrm{A}$ dissections and 15 patients with acute type $\mathrm{B}$ dissections.

With the advantages of minimally invasive procedures, TEVAR plays an important role, especially in older patients with multiple co-morbidities suffering from acute type A aortic dissection (ATAAD). Complete coverage of the entry tear is essential in the endovascular intervention of ATAAD as it depressurizes the false lumen, redirecting blood flow into the true lumen, resulting in thrombosis of the false lumen, thus allowing for aortic remodeling.

Nienaber et al. ${ }^{[3]}$ reported an international multi-centre experience of 12 type A aortic dissection (TAAD) patients ( 6 cases of acute and subacute or chronic each) with a mean age of $81 \pm 7$ years and a Euro SCORE

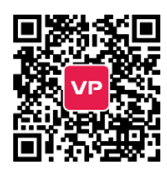


II $9.1 \pm$ 4.5. These patients received endovascular therapy using ZENITH TX2 (Cook, Bloomington, Ind), GORE C-Tag (Gore Ltd., London, United Kingdom), or Relay NBS (Bolton, Barcelona, Spain). The early results revealed a technical success rate of $91.7 \%$, all with proper aortic remodeling and low complication rates ( 1 transient stroke and 1 death due to wire induced perforation). Follow-up CT scans demonstrated good aortic remodeling. In 2020, Ghoreishi et al. ${ }^{[4]}$ reported a single-centre experience of 13 patients with ascending aorta disease and a mean age of $69 \pm 9$ years (including 7 patients with ATAAD) using Gore TAG (CTAG) (W. L. Gore \& Associates, Flagstaff, Ariz). 100\% technical success (2 with location of zone 0a) was achieved and proper aortic remodeling was found on follow-up CT scans. Both studies revealed excellent results in the elderly and patients with comorbidities with TAAD.

In general, the procedure of TEVAR consists of the following steps ${ }^{[3,4]}:(1)$ a temporary pacing wire is inserted to the right ventricle via venous cut-down; (2) a pigtail catheter to the left ventricle is navigated using a soft guide wire through the true lumen under imaging guidance to confirm the location; (3) a soft guide wire is then exchanged to a stiff guide wire, followed by delivery of the endograft device to the ascending aorta under rapid ventricular pacing to reduce the windsock effect during graft deployment; (4) the location of stent-graft, the patency of coronary arteries and arch vessel, the presence of endoleaks, and the occurrence of aortic regurgitation are evaluated by angiogram; and (5) the pigtail catheter and temporary pacing wire are retracted and access sites closed.

Nonetheless, issues have emerged from the current status of endovascular repair for ATAAD based on unmet requirements.

\section{TECHNICAL ASPECTS OF THE PROCEDURE: A REVIEW OF THE LITERATURE}

One issue identified is the immediate impact upon deployment of the stent. The lack of specially designed stents for the ascending aorta not only increases difficulties of the procedure and patient selection, but also raises concerns of the unknown effects that the stent-graft has on the ascending aorta in the multiply comorbid patient with poor cardiovascular reserve. In previous studies, it has been reported that endovascular device made of artificial material was of poor compliance and even Dacron polyester fabric grafts were four times less compliant compared to native arteries ${ }^{[5,6]}$.

Additionally, elderly patients with multiple comorbidities are pro-inflammatory, which leads to systemic microvascular endothelial inflammation with subsequent myocardial inflammation and fibrosis. This increases oxidative stress and alternation in cardiomyocyte signaling pathways which promotes cardiac remodeling and dysfunction. Therefore, it is crucial to explore an ideal stent-graft, which alleviates the harmful long-term effects and potential pathogenicity to these susceptible patients ${ }^{[7]}$.

Anatomical complexities are another issue surgeons face. Following ATAAD, ascending aorta dilatation, arch dilatation and aortic valve insufficiency can occur, and the walls of the aorta become fragile. In order to achieve proper fixation and seal of the stent graft, adequate length of landing zone in both proximal and distal site of the stent is essential. Proximally, the serrated edge of the stent may cover the coronary orifices or interfere with the commissures of the aortic valve, resulting in an entry tear in the proximal third of the aorta close to the sinus-tubular junction or even the sinus portion. Roselli et al ${ }^{[8]}$ proposed a modified classification of landing zones, dividing the ascending aorta into zones $\mathrm{OA}, \mathrm{OB}$ and $\mathrm{OC}$ to address the anatomical complexity and importance ${ }^{[8]}$. Diseases extending to zone $0 \mathrm{~A}$ pathology (from the annulus to the distal margin of the highest coronary) had significantly worse outcomes than others. Distally, Sobocinski et al. ${ }^{[9]}$ analyzed the feasibility of endovascular therapy and revealed that it may be acceptable to expand the margin of the distal landing zone from the additional debranching of the brachiocephalic trunk to the left common carotid artery. Of note, orifices of the innovate artery, left common carotid artery and left subclavian artery are in close proximity, which gives us limited choice of commercial stent-grafts 
because these grafts are too long for the ascending aorta to obtain a sufficient landing zone. Thus, it is important to have specially designed stent-grafts and accurate imaging study before the procedure.

To date, there still remains some controversy surrounding patient selection for TEVAR. Conventionally, aortic valve insufficiency is a contraindication for TEVAR ${ }^{[10]}$, however, more than one-third of ATAAD patients, especially in the severe subgroup, were found to have this. In 2014, Rylski et al. ${ }^{[11]}$ introduced the concept of endovascular treatment of ascending aortic pathologies with valve-carrying conduits associated with an uncovered portion for free diastolic coronary blood flow ${ }^{[11]}$. Using an endovascular valve-carrying conduit not only resolves the problem of aortic regurgitation, but also effectively results in sufficient anchorage of the device ${ }^{[12]}$. Alternatively, Nienaber et al. ${ }^{[3]}$ proposed a combined TAVR (transcatheter aortic valve replacement)-TEVAR technology, in an attempt to treat variants of aortic dissection including those with compromised aortic valve function.

Fatal complications such as aortic rupture ${ }^{[2]}$, ventricular perforation and cardiac tamponade ${ }^{[3]}$, or other early morbidities such as supraventricular tachycardia and cardiovascular ischemia have been reported in the current literature. This also raises the concern of cerebrovascular accidents that may occur as the vascular surgeon passes the guide wire and deploys the stent-graft in a calcified ascending aorta and arch of ATAAD patients with advanced age and multiple morbidities. In addition, unidentified acute coronary involvement (ACI) in ATAAD would be fatal and it is worth noting that up to $30 \%$ of patients with ACI disclosed no clinical manifestations of coronary malperfusion preoperatively ${ }^{[13]}$.

Once stent implantation is successfully achieved, the second issue is its delayed impact on aortic stiffness. A healthy aorta has a cushioning function, limiting arterial pulsatility and protects the microvasculature from potentially harmful fluctuations in pressure and blood flow ${ }^{[14]}$. With a complex structure close to the left ventricle (LV), any deviation from the natural physiologic character of the ascending aorta (such as increased aorta stiffness) would give rise to complications. Available stent-grafts made of artificial compounds, such as expanded polytetrafluoroethylene and woven polyester, and metal wire, are poorly compliant and foreign to the human body. Current Dacron polyester fabric grafts exhibit four times reduced compliance compared to native arteries ${ }^{[5,6]}$. Hence, it is reasonable to assume that wire-containing stent-grafts are less compliant ${ }^{[5]}$. An immobilized segment is created after placement of the stent-graft in the ascending aorta, alternating in diameter and area during every heartbeat, and more aortic stiffness develops consequently. Without enough elasticity, the Windkessel effect vanishes leading to more resistance to LV and rebound force on the aortic valve. It is well established that large-artery stiffness (LAS) impairs the aortic cushioning function and independently predicts cardiovascular risk. LAS also contributes to isolated systolic hypertension, excessive penetration of pulsatile energy into the microvasculature of target organs that operate at low vascular resistance, and abnormal ventricular-arterial interactions that promote left ventricular remodeling, dysfunction, and failure ${ }^{[14]}$. In a 4 -year follow-up retrospective study using patient-specific fluid-structure interaction analysis and image-based measurements of cardiac remodeling from echocardiography and computed tomography angiography, van Bakel et al. ${ }^{[15]}$ demonstrated that TEVAR-induced acute aortic stiffening caused a $26 \%$ increase in LV stroke work and cardiac remodeling ${ }^{[15]}$. Moreover, alternated left ventricular hemodynamics may raise concerns of impaired function of the LV induced by ventricular remodeling and impairment of the aortic valve. It has been found that aortic stiffness is associated with LV remodeling and reduced LV systolic and diastolic function by magnetic resonance imaging measurement in a large multi-ethnic population ${ }^{[16]}$. Therefore, it is crucial to explore an ideal stent-graft so as to alleviate the harmful long-term effects and potential pathogenicity to these susceptible patients. In addition, a thorough examination of cardiac morphology and function should be performed ${ }^{[5]}$ in the long-term follow up including echocardiographic parameters of (1) key structural alterations, such as the left atrial volume index or left ventricular mass index and (2) key functional alterations, such as an E/e' ratio of early mitral inflow velocity and an $\mathrm{E} / \mathrm{A}$ denoting ratio of $\mathrm{E}$ wave to $\mathrm{A}$ wave ${ }^{[17]}$. 


\section{FINAL REMARKS}

Reports from the International Registry of Acute Aortic Dissections show that 10\% to 30\% of ATAAD patients are considered too high risk to receive open repair and would therefore receive only medical management with up to $60 \%$ early mortality rates ${ }^{[3,18]}$. From an anatomical aspect, based on valvular condition, landing zone, and coronary involvement, 30\% to 50\% of patients are technically suitable for TEVAR $^{[19]}$. To date, ascending TEVAR strategies appear encouraging in the treatment of various ascending aortic pathologies. Thus, ascending TEVAR is feasible and reveals promising early and short-term results based on our updated literature review [Supplementary Table 1]. Nevertheless, the complexity of the anatomy in the ascending aorta continues to be a major obstacle for the use of current endovascular technologies. In other words, ascending TEVAR is often highly compromised by anatomic limitations with the short length of the ascending aorta, the location of the coronary ostia, the location of the entry tear, and the supra-aortic branches ${ }^{[13]}$. Of them, the most common criteria contradictory for ascending TEVAR was the lack of a sufficient landing zone (i.e., the distance between the coronary ostia and the entry tear has been measured to be less than $\left.20 \mathrm{~mm}^{[13,20]}\right)$. Notably, Roselli et al ${ }^{[8]}$ pointed out that the greater curve is more than $30 \%$ longer than the lesser curve along the length of the ascending aorta ${ }^{[8]}$. Thus, the nextgeneration ascending stent-graft device needs to be highly conformable and elastic with adequate strength of fixation in what is a hostile environment ${ }^{[8]}$. In summary, there are no commercially available designs of endovascular devices specifically for the ascending aorta currently. The current iteration of stent-graft technology however, needs to be adapted to the specific anatomic features of the ascending aorta ${ }^{[3]}$. It is envisioned that with time, combining new knowledge and technological advancements will further pave the way for broader application of ascending TEVAR for patients with TAAD.

\section{DECLARATIONS}

\section{Authors' contributions}

Authors made substantial contributions to the conception and design of the study, and participated in drafting the article: Chang TW, Yang TT

Authors provided administrative, technical, and material support: Jhou HJ, Ke LY

The author participated in critical revision for important conceptual and intellectual content and gave final approval of the version to be submitted to the Journal: Chen YF

\section{Availability of data and materials}

Not applicable.

\section{Financial support and sponsorship}

None.

\section{Conflicts of interest}

All authors declared that there are no conflicts of interest.

\section{Ethical approval and consent to participate}

Not applicable.

\section{Consent for publication}

Not applicable.

\section{Copyright}

(c) The Author(s) 2020. 


\section{REFERENCES}

1. Czerny M, Schmidli J, Adler S, van den Berg JC, Bertoglio L, et al. Current options and recommendations for the treatment of thoracic aortic pathologies involving the aortic arch: an expert consensus document of the European Association for Cardio-Thoracic Surgery (EACTS) \& the European Society for Vascular Surgery (ESVS). Eur J Cardiothorac Surg 2019;55:133-62.

2. Dake MD, Kato N, Mitchell RS, Semba CP, Razavi MK, et al. Endovascular stent-graft placement for the treatment of acute aortic dissection. N Engl J Med 1999;340:1546-52.

3. Nienaber CA, Sakalihasan N, Clough RE, Aboukoura M, Mancuso E, et al. Thoracic endovascular aortic repair (TEVAR) in proximal (type A) aortic dissection: Ready for a broader application? J Thorac Cardiovasc Surg 2017;153:S3-11.

4. Ghoreishi M, Shah A, Jeudy J, Pasrija C, Lebowitz J, et al. Endovascular repair of ascending aortic disease in high-risk patients yields favorable outcome. Ann Thorac Surg 2020;109:678-685.

5. Yang TT, Chang TW, Chen YF. Thoracic endovascular aortic repair in type A aortic dissection: Exciting results with unsolved problems. J Thorac Cardiovasc Surg 2017;154:1220-1.

6. Spadaccio C, Nappi F, Al-Attar N, Sutherland FW, Acar C, et al. Old myths, new concerns: the long-term effects of ascending aorta replacement with dacron grafts. Not all that glitters is gold. J Cardiovasc Transl Res 2016;94:334-42.

7. Redfield MM. Heart failure with preserved ejection fraction. N Engl J Med 2016;375:1868-77.

8. Roselli EE, Idrees JJ, Johnston DR, Eagleton MJ, Desai MY, et al. Zone zero TEVAR: a proposed modification to the classification of landing zones. J Thorac Cardiovasc Surg 2018;155:1381-9.

9. Sobocinski J, O’Brien N, Maurel B, Bartoli M, Goueffic Y, et al. Endovascular approaches to acute aortic type A dissection: a CT-based feasibility study. Eur J Vasc Endovasc Surg 2011; 42:442-7.

10. Rylski B, Bavaria JE, Milewski RK, Vallabhajosyula P, Moser W, et al. Long-term results of neomedia sinus valsalva repair in 489 patients with type A aortic dissection. Ann Thorac Surg 2014;98:582-8.

11. Rylski B, Szeto WY, Bavaria JE, Branchetti E, Moser W, et al. Development of a single endovascular device for aortic valve replacement and ascending aortic repair. J Card Surg 2014;29:371-6.

12. Kreibich M, Rylski B, Kondov S, Morlock J, Scheumann J, et al. Endovascular treatment of acute type A aortic dissection-the endo bentall approach. J Vis Surg 2018;4;4:69.

13. Chen YF, Chien TM, Yu CP, Ho KJ, Wen H, et al. Acute aortic dissection type A with acute coronary involvement: a novel classification. Int J Cardiol 2013;168:4063-9.

14. Chirinos JA, Segers P, Hughes T, Townsend R. Large-artery stiffness in health and disease. J Am Coll Cardiol 2019;74;1237-63.

15. van Bakel TMJ, Arthurs CJ, Nauta FJH, Eagle KA, van Herwaarden JA, et al. Cardiac remodelling following thoracic endovascular aortic repair for descending aortic aneurysms. Eur J Cardiothorac Surg 2019;1;55:1061-70.

16. Ohyama Y, Ambale-Venkatesh B, Noda C, Chugh AR, Teixido-Tura G, et al. Association of aortic stiffness with left ventricular remodeling and reduced left ventricular function measured by MRI: the multi-ethnic study of atherosclerosis. Circ Cardiovasc Imaging 2016;9:e004426.

17. Ponikowski P, Voors AA, Anker SD, Bueno H, Cleland JGF, et al. 2016 ESC guidelines for the diagnosis and treatment of acute and chronic heart failure: the task force for the diagnosis and treatment of acute and chronic heart failure of the european society of cardiology (ESC) developed with the special contribution of the heart failure association (HFA) of the ESC. Eur Heart J 2016;37:2129-200.

18. Hagan PG, Nienaber CA, Isselbacher EM, Bruckman D, Karavite DJ, et al. The international registry of acute aortic dissection (IRAD): new insights into an old disease. JAMA 2000;283:897-903.

19. Moon MC, Greenberg RK, Morales JP, Martin Z, Lu Q, et al. Computed tomography-based anatomic characterization of proximal aortic dissection with consideration for endovascular candidacy. J Vasc Surg 2011;53:942-9.

20. Roselli EE, Idrees J, Greenberg RK, Johnston DR, Lytle BW. Endovascular stent grafting for ascending aorta repair in high-risk patients. J Thorac Cardiovasc Surg 2015;149:144-51. 